\title{
ON STRONGLY REGULAR NEAR-RINGS
}

\author{
by Y. V. REDDY and C. V. L. N. MURTY
}

(Received 19th April, 1983)

\section{Introduction}

According to Mason [1] a right near-ring $N$ is called (i) left (right) strongly regular if for every $a$ there is an $x$ in $N$ such that $a=x a^{2}\left(a=a^{2} x\right)$ and (ii) left (right) regular if for every $a$ there is an $x$ in $N$ such that $a=x a^{2}\left(a=a^{2} x\right)$ and $a=a x a$. He proved that for a zerosymmetric near-ring with identity, the notions of left regularity, right regularity and left strong regularity are equivalent. The aim of this note is to prove that these three notions are equivalent for arbitrary near-rings. We also show that if $N$ satisfies dcc on $N$-subgroups, then all the above four notions are equivalent.

In what follows $N$ stands for a right near-ring. For notation and basic results, we shall refer to [2].

We first prove a couple of lemmas.

Lemma 1. Suppose $N$ is a left strongly regular near-ring. If $a b=0$ for some $a, b$ in $N$, then $b a=b 0$.

Proof. Since $a b=0$, we have that $(b a)^{2}=b 0 a=b 0$. So, it is enough if we show that $b a$ $=(b a)^{2}$. Since $N$ is left strongly regular, there is an element $x \in N$ such that $b a=x(b a)^{2}$. So, $b a=x b 0$ and hence $(b a)^{2}=(b a)(b a)=(x b 0)(b a)=x b 0=b a$.

Lemma 2. Suppose $N$ is a left strongly regular near-ring. If $b^{2}=b^{3}$ for some $b \in N$, then $b=b^{2}$.

Proof. Since $N$ is left strongly regular, we can find $y$ in $N$ such that $b=y b^{2}$. Then $h=y h^{2}=y b h=y\left(y b^{2}\right) b=y^{2} b^{3}=y^{2} b^{2} \quad$ (by hypothesis) and so, $b=y\left(y b^{2}\right)=y b$. Hence $h^{2}=y b^{2}=b$.

Theorem 3. Let $N$ be a left strongly regular near-ring. If $a=x a^{2}$ for some $a, x$ in $N$, then $a=a x a$ and $a x=x a$.

Proof. (i) Since $a=x a^{2},(a-a x a) a=0$ and hence by Lemma $1, a(a-a x a)=a 0$ and $a \times a(a-a x a)=a \times a 0$. Therefore $(a-a \times a)^{2}=a(a-a \times a)-a \times a(a-a x a)=a 0-a \times a 0=(a 0$ $-a x) a 0$. Now $(a-a x a)^{3}=(a 0-a x) a 0(a-a x a)=(a 0-a x) a 0=(a-a x a)^{2}$. So, by Lemma 2 we have $(a-a x a)^{2}=a-a x a$. Consequently $0=(a-a x a) a=(a-a x a)^{2} a=(a 0-a x) a 0 a$ $=(a 0-a x) a 0=(a-a x a)^{2}=a-a x a$. Thus $a=a x a$. (ii) Now clearly, $(a x-x a) a=0$. As in (i), we can prove that $a x-x a=(a x-x a)^{2}=a x 0-x a 0$. Therefore $0=(a x-x a) a=(a x$ $-x a) 0 a=(a x-x a) 0=a x-x a$. Thus $a x=x a$. 
Corollary 4. A near-ring $N$ is left strongly regular if and only if it is left regular.

Proof. If $N$ is left strongly regular, then for each $a \in N$, there exists $x \in N$ such that $a=x a^{2}$. It follows from Theorem 3 that $a=a x a$. Therefore $N$ is left regular. The converse is obvious.

The proof of the following corollary follows directly from Theorem 3 .

Corollary 5. If $N$ is left strongly regular, then it is right regular.

Corollary 6 ([1], Lemma 2). A left regular near-ring with IFP is right regular.

Corollary 7 ([1], Proposition 1). If $N$ is zerosymmetric, then left regularity is equivalent to left strong regularity and these imply right regularity.

Before proving the equivalence of the three notions of left regularity, right regularity and left strong regularity, we introduce the following definition.

Definition 8. We say that a near-ring $N$ has the property $\left(^{*}\right)$ if it satisfies:

(i) for any $a, b$ in $N, a b=0$ implies $b a=b 0$.

(ii) for any $a$ in $N, a^{2}=a^{3}$ implies $a=a^{2}$.

Remark. From Lemmas 1 and 2, it follows that every left strongly regular near-ring has the property $\left({ }^{*}\right)$.

Lemma 9. Suppose $N$ is a right strongly regular near-ring. Then $N$ has the property $(*)$.

Proof. (i) Let $a, b \in N$ with $a b=0$. Then there exists $x \in N$ such that $b a=(b a)^{2} x$ and so, $b a=b 0 a x=b 0$.

(ii) Let $a \in N$ with $a^{2}=a^{3}$. Then $a=a^{2} x$ for some $x \in N$. Hence $a^{2}=a\left(a^{2} x\right)=a^{3} x=a^{2} x$ $=a$.

Theorem 10. If $N$ has the property $\left({ }^{*}\right)$, then for any idempotent $e$ and any $n$ in $N$, en $=$ ene.

Proof. Let $e^{2}=e$ and $n \in N$. Clearly (en-ene) $e=0$. As in the proof of Theorem 3, we can prove that $e n-e n e=(e n-e n e)^{2}=e n 0-e n e 0$. Hence $0=(e n-e n e) e=e n-e n e$. Thus en $=$ ene.

The proof of the following corollary follows directly from Lemmas 1, 2, 9 and Theorem 10.

Corollary 11. If $N$ is either a left or right strongly regular near-ring, then for any idempotent $e$ and any $n$ in $N$, en=ene. 
Recall that a near-ring $N$ is called regular if for each $a$ in $N$, there exists $x$ in $N$ such that $a=a x a$.

Theorem 12. The following statements are equivalent:

(i) $N$ is left strongly regular.

(ii) $N$ is right regular.

(iii) $N$ is regular and en=ene for any idempotent $e$ and any $n$ in $N$.

Proof. (i) $\Rightarrow$ (ii) follows from Corollary 5 .

(ii) $\Rightarrow$ (iii): If $N$ is right regular then, by definition, it is right strongly regular. Now the result follows from Corollary 11.

(iii) $\Rightarrow$ (i): Let $a \in N$. Since $N$ is regular, $a=a x a$ for some $x \in N$. Since $x a$ is an idempotent by our assumption, $a x=(a x a) x=a(x a) x=\operatorname{axaxx} a=y a$, where $y=a \times a x x$ and hence $a=a x a=y a^{2}$. Thus $N$ is left strongly regular.

Combining Corollary 4 and Theorem 12, we have the following:

Proposition 13. The following statements concerning an arbitrary near-ring $N$ are equivalent:

(i) $N$ is left strongly regular.

(ii) $N$ is right regular.

(iii) $N$ is left regular.

Corollary 14 ([1], Proposition 1). For a zerosymmetric unital near-ring the notions of left regularity, left strong regularity and right regularity are all equivalent.

Theorem 15. Suppose $N$ satisfies dcc on $N$-subgroups of $N$. Then $N$ is right strongly regular if and only if it is left strongly regular.

Proof. Assume that $N$ is right strongly regular. Let $a \in N$. Consider the chain $N a \supseteq N a^{2} \supseteq N a^{3} \supseteq \ldots$ Since $N$ has dcc on $N$-subgroups, there exists $n>1$ such that $N a^{n}=N a^{n+1}=N a^{n+2}=\ldots$. So, $a^{n+1}=x a^{n+2}$ for some $x \in N$. Now $\left(a^{n}-x a^{n+1}\right) a^{n}=0$. Using Lemma 9, as in the proof of Theorem 3, we can prove that $a^{n}-x a^{n+1}=\left(a^{n}-x a^{n+1}\right)^{2}$ $=a^{n} 0-x a^{n+1} 0$. Hence $0=\left(a^{n}-x a^{n+1}\right) a^{n}=\left(a^{n} 0-x a^{n+1} 0\right) a^{n}=a^{n} 0-x a^{n+1} 0=a^{n}-x a^{n+1}$. Thus $a^{n}=x a^{n+1}$. Continuing in this way, we can show that $a=x a^{2}$. Thus $N$ is left strongly regular. The converse follows from Theorem 3.

The following corollary is a direct consequence of Theorems 12 and 15.

Corollary 16. If $N$ satisfies $d c c$ on $N$-subgroups of $N$, then the following are equivalent: (i) $N$ is left regular, (ii) $N$ is right regular, (iii) $N$ is left strongly regular, (iv) $N$ is right strongly regular. 
Acknowledgement. The second author wishes to express thanks to the Council of Scientific and Industrial Research, New Delhi for giving financial assistance.

\section{REFERENCES}

1. G. Mason, Strongly regular near-rings, Proc. Edinburgh Math. Soc. 23 (1980), 27-35.

2. G. PiLz, Near-rings (North-Holland, Amsterdam, 1977).

Department of Mathematics

NagarJuna University

NagarjunANAGar- 522510

A.P. INDIA 